\title{
V/P SPECT as a diagnostic tool for pregnant women with suspected pulmonary embolism
}

\author{
Marika Bajc $^{1} \cdot$ Berit Olsson $^{1} \cdot$ Anders Gottsäter $^{3} \cdot$ Cecilia Hindorf $^{2} \cdot$ Jonas Jögi $^{1}$
}

Received: 9 January 2015 / Accepted: 18 March 2015 / Published online: 28 April 2015

(C) The Author(s) 2015. This article is published with open access at Springerlink.com

\begin{abstract}
Purpose The purpose of the study was to assess the prevalence of pulmonary embolism (PE) and other lung diseases among pregnant women with suspected PE and to calculate the radiation exposure to patient and fetus in this population. As a secondary aim, we evaluated the negative predictive value of a normal ventilation/perfusion single photon emission computed tomography (V/P SPECT) examination in pregnancy.

Methods We studied all 127 pregnant women who had suspected PE and had undergone V/P SPECT at our institution in the course of a 5-year period. Radiation exposure to patient and fetus and the negative predictive value of a normal V/P SPECT examination were also measured.

Results V/P SPECT identified PE in 11 women (9\%). Moreover, in 15 women (12\%) the examination revealed pneumonia (in 2 cases in addition to PE) and in 1 woman signs of airway obstruction were revealed. Among the 116/ 127 women (91 \%) where PE was ruled out by V/P SPECT, none was diagnosed subsequently with PE or deep venous thrombosis (DVT) during the same pregnancy or puerperal period. For P SPECT, the calculated fetal absorbed dose was $<0.6 \mathrm{mGy}$, and the calculated breast absorbed dose $0.6 \mathrm{mGy}$. For V SPECT, the calculated fetal absorbed dose was $<$ $0.014 \mathrm{mGy}$ and the breast absorbed dose $0.25 \mathrm{mGy}$.
\end{abstract}

Marika Bajc

marika.bajc@med.lu.se

1 Clinical Physiology and Nuclear Medicine, Skåne University Hospital and Lund University, 22185 Lund, Sweden

2 Radiation Physics, Skåne University Hospital, Lund, Sweden

3 Vascular Diseases, Skåne University Hospital, Malmö, Sweden
Conclusion The prevalence of PE was low (9\%) among pregnant women with suspected disease. Pneumonia was diagnosed in $12 \%$ of patients. The negative predictive value of V/P SPECT was high, and the radiation exposure from V/P SPECT was low both for fetus and patient.

Keywords Pregnancy · Pulmonary embolism - V/P SPECT . Radiation exposure

\section{Introduction}

The risk for pulmonary embolism (PE) is increased about fivefold during pregnancy and the puerperal period $[1,2]$ due to both changes in the coagulation system and mechanical factors such as vein compression, and more than $50 \%$ of events occur in the first 20 weeks of pregnancy [3]. As clinical symptoms are unspecific, it is important to take into account the possibility of $\mathrm{PE}$ when a pregnant woman experiences symptoms such as chest pain, dyspnoea, pyrexia, tachycardia, leg pain and swelling (particularly in the left leg) or lower abdominal pain. However, many of these symptoms may be due to the physiological stress of pregnancy or to other cardiopulmonary diseases, such as pneumonia or on rare occasions left heart failure. In the initial diagnostic evaluation of $\mathrm{PE}$, testing of clinical probability is important, but few of the test algorithms have been evaluated during pregnancy [4]. Ddimer is usually increased during pregnancy, and therefore current evidence does not support the use of negative Ddimer to rule out PE in a pregnant woman [5]. Therefore, a clinical suspicion of PE always needs to be confirmed by an imaging test. Currently, ventilation/perfusion single photon emission computed tomography (V/P SPECT) $[6,7]$ or planar pulmonary scintigraphy [8] is recommended by different European guidelines as an initial imaging modality, based on 
its low radiation exposure, high sensitivity and specificity and possibility for follow-up. The American Society of Thoracic Radiology [9] also recommends planar pulmonary scintigraphy as the preferred imaging method during pregnancy.

We studied all pregnant women who underwent V/P SPECT at our institution for suspected PE over a 5-year period. The primary aims of the study were to assess the prevalence of PE and other lung diseases and to calculate the radiation exposure to patient and fetus in this population. As a secondary aim, we evaluated the negative predictive value of a normal V/P SPECT examination in pregnancy.

\section{Materials and methods}

In the course of a 5-year period (2009-2013), 127 pregnant women (mean age 30 years, range 18-48) were referred for examination with V/P SPECT due to suspected PE at Skåne University Hospital, Lund, Sweden. During the study period, 30 patients $(24 \%)$ were examined in the first trimester (weeks $1-15), 59(46 \%)$ in the second trimester (weeks 16-27) and $38(30 \%)$ in the third trimester (weeks 28-40). In accordance with the European guidelines $[6,7]$, the 2-day protocol was implemented during the first trimester of pregnancy: P SPECT only on the first day and V SPECT performed the next day only if indicated (Table 1). Chest X-ray was performed only if considered clinically indicated. Patient files from all hospitals in the region were checked for potential later PE or deep venous thrombosis (DVT) diagnoses made in the 127 women during the same pregnancy and puerperal period. Clinical characteristics and symptoms at presentation in the 127 women are shown in Table 2.

\section{Absorbed dose calculation}

Breast doses were calculated using data from ICRP 53 and ICRP $80[10,11]$ and fetal doses were calculated according to Russell et al. [12]. The ${ }^{99 \mathrm{~m}} \mathrm{Tc}-\mathrm{Tech}$ egas was not assumed to cross over to the placenta, which means that the only contribution to the fetal dose is the radiation from activity within the lungs of the mother. The specific absorbed fractions for photons were obtained from www.doseinfo-radar.com, accessed on 23 Apr 2014, and were recalculated to the photon energy

Table 1 Different examination techniques used in 127 pregnant women undergoing V/P SPECT for suspected PE

\begin{tabular}{lllll}
\hline Trimester & Patients & P SPECT & V SPECT & V/P SPECT \\
\hline 1st (weeks 1-15) & 30 & 14 & 13 & 3 \\
2nd (weeks 16-27) & 59 & 6 & 1 & 52 \\
3rd (weeks 28-40) & 38 & & & 38 \\
\hline
\end{tabular}

Data shown separately for women in each trimester of pregnancy
Table 2 Clinical characteristics and symptoms at presentation $[n(\%)]$ in 127 pregnant women undergoing V/P SPECT for suspected PE

\begin{tabular}{ll}
\hline Clinical characteristics & $n(\%)$ \\
\hline Previous VTE & $6(5)$ \\
Family history of VTE & $12(9)$ \\
Pneumonia & $7(6)$ \\
Bronchial asthma or chronic obstructive pulmonary disease & $8(6)$ \\
Pulmonary histiocytosis & $1(1)$ \\
Cystic fibrosis & $1(1)$ \\
Pre-eclampsia & $1(1)$ \\
Symptoms & \\
Chest pain & $52(41)$ \\
Dyspnoea & $69(54)$ \\
Cough & $15(12)$ \\
Syncope & $3(2)$ \\
High fever & $2(2)$ \\
Haemoptysis & $6(5)$ \\
Swollen leg & $7(6)$ \\
\hline
\end{tabular}

$V T E$ venous thromboembolism

for ${ }^{99 \mathrm{~m}} \mathrm{Tc}(140 \mathrm{keV})$. The ${ }^{99 \mathrm{~m}} \mathrm{Tc}-\mathrm{Technegas}$ was assumed to remain within the lungs until complete decay as no biological excretion of the radiopharmaceutical occurs.

\section{Perfusion (P) SPECT}

P SPECT was performed in accordance with the guidelines of the European Association for Nuclear Medicine (EANM) [6, 7] with a dual-head gamma camera in the supine position, after i.v. administration of $50 \mathrm{MBq}{ }^{99 \mathrm{~m}} \mathrm{Tc}$-macroaggregated albumin (MAA, TechneScan LyoMAA ${ }^{\circledR}$, Mallinckrodt Medical, Petten, The Netherlands).

\section{Ventilation (V) SPECT}

V SPECT was performed after inhalation of aerosolized ${ }^{99 \mathrm{~m}}$ Tc-Technegas ${ }^{\circledR}$ (Cyclomedica Ltd, Lucas Heights, NSW, Australia), reaching $30 \mathrm{MBq}$ in the lungs. The procedure was carried out with the patient in the supine position over a period of $11 \mathrm{~min}$ ( $10 \mathrm{~s}$ per stop, 64 stop for each camera) as described elsewhere [13].

\section{V/P SPECT protocol}

The 1-day protocol for V/P SPECT started with inhalation of up to $30 \mathrm{MBq}{ }^{99 \mathrm{~m}} \mathrm{Tc}$-Technegas to the lung. V SPECT was performed in the supine position, with acquisition lasting 11 min. Without changing the patient position, P SPECT followed immediately, after i.v. injection of $120 \mathrm{MBq}{ }^{99 \mathrm{~m}} \mathrm{Tc}$ MAA, with acquisition lasting $5 \mathrm{~min}$. The methodology has been described in full elsewhere $[13,14]$. Over the same 5- 
year period, 61 pregnant women with suspected PE were examined with multidetector CT (MDCT), 37 (61\%) of them during the night or weekends when V/P SPECT was not available.

\section{Statistics and ethics}

The study is a descriptive analysis, which had been approved by the local Ethics Committee of Lund University.

\section{Results}

PE was identified using V/P SPECT in 11 (9\%) of the 127 patients. The extent of PE was small to medium, not exceeding $40 \%$ of the pulmonary vascular bed in any case. Moreover, in 15 patients (12\%) the examination revealed pneumonia [7] (in 2 of these cases in addition to PE), and in 1 patient it indicated signs of airway obstruction, defined in [15] (Table 3). Among the 30 patients examined during the first trimester, PE was diagnosed in 5. In this group, 14 patients underwent P SPECT only and only 1 in this group was diagnosed with PE, whereas 13 patients underwent V SPECT the next day and among them 4 were diagnosed with PE, 1 with concomitant pneumonia. In four patients pneumonia was observed as the sole finding. Among patients examined during the second trimester, 4 of 59 were diagnosed with PE. Six patients underwent $\mathrm{P}$ SPECT only and none was positive for PE. One more patient needed a V SPECT the next day which showed findings typical for pneumonia. Among 52 other patients undergoing the 1-day V/P SPECT protocol 4 were diagnosed with PE, 1 with concomitant pneumonia (Fig. 1) that was also clinically treated. Moreover, one woman was diagnosed with airway obstruction.

Among those examined during the last trimester, 3 of 38 women were diagnosed with PE, 1 of whom also had pneumonia. In four other women, signs of pneumonia were observed. Among the 116 of 127 patients (91\%) in whom PE was ruled out by V/P SPECT, none was diagnosed with PE or

Table 3 Results of P and V SPECT in 127 pregnant women undergoing V/P SPECT for suspected PE

\begin{tabular}{lllll}
\hline & & \multicolumn{3}{l}{ Trimester } \\
\cline { 3 - 5 } & & 1 & 2 & 3 \\
\hline PE & $11(9 \%)$ & 5 & 4 & 2 \\
Pneumonia & $15(12 \%)$ & 4 & 6 & 5 \\
Left heart failure & $1(<1 \%)$ & & 1 & \\
Airway obstruction & $1(<1 \%)$ & & 1 & \\
\hline
\end{tabular}

Results shown both in total and separately for women in each trimester of pregnancy
DVT during the same pregnancy or the puerperal period. A negative study was thus $100 \%$ predictive of no clinically apparent emboli in this time period.

Calculated breast absorbed doses were $0.2 \mathrm{mGy}$ after inhalation of $30 \mathrm{MBq}$ of ${ }^{99 \mathrm{~m}} \mathrm{Tc}-$ Technegas and $0.6 \mathrm{mGy}$ after intravenous injection of $120 \mathrm{MBq}{ }^{99 \mathrm{~m}} \mathrm{Tc}-\mathrm{MAA}$. If only $\mathrm{P}$ SPECT was performed calculated breast absorbed dose was $0.25 \mathrm{mGy}[10-12]$. The doses to the fetus were calculated to the stage of gestation (Table 4) [10-12].

\section{Multidetector computed tomography}

Among pregnant women undergoing MDCT during the 5year period, 3 of 61 showed signs of PE. In two of these, the examination was not considered as optimal. However, six patients showed parenchymal changes, and in six other cases the examination was reported by the radiologist as inconclusive and not allowing exclusion of PE. One patient underwent two MDCT examinations that were both considered inconclusive. Among 127 pregnant women, 7 were suspected for DVT. Five were examined with ultrasound, and only one was pathological.

\section{Discussion}

This is the first study reporting the value of V/P SPECT during pregnancy. It established that we were able to follow the European guidelines [6, 7] and use the proposed 2-day protocol for PE diagnosis in the first trimester of pregnancy in the majority of women in this group. Moreover, V/P SPECT was demonstrated to be a safe procedure regarding sensitivity, negative predictive value and radiation exposure to the fetus and the pregnant woman. All reports were diagnostic. P SPECT alone was sufficient in 20 patients and identified women in whom it was necessary to perform the additional V SPECT to confirm or exclude PE and comorbidity. Furthermore, V SPECT helped to clarify the cause of symptoms by identifying additional diagnoses of pneumonia and chronic obstructive pulmonary disease (COPD). The majority of our patients thus benefited from V SPECT.

The prevalence of PE among pregnant women with suspected disease was highest during the first trimester, confirming results from other investigators [2]. However, the prevalence of $\mathrm{PE}$ in our study during the entire pregnancy was $9 \%$, which is higher than presented by others $[16,17]$. The reason for this may be that previous investigators might have underestimated the true incidence either by not sending patients for imaging tests or alternatively by relying on MDCT which in the PIOPED II study [18] showed a comparably low sensitivity for PE diagnosis in the general population. Furthermore, Ridge et al. reported a high number of nondiagnostic findings on MDCT during pregnancy and therefore 
Fig. 1 Frontal (a) and sagittal (b) slices from V/P SPECT and chest $\mathrm{X}$-ray in a pregnant woman diagnosed with both PE and pneumonia in the second trimester of pregnancy. Red arrows indicate segmental perfusion defects in areas with preserved ventilation representing $\mathrm{PE}$ in the right and left lung. Blue arrows indicate absent ventilation and reduced perfusion representing pneumonia in the lower left lung a

Frontal

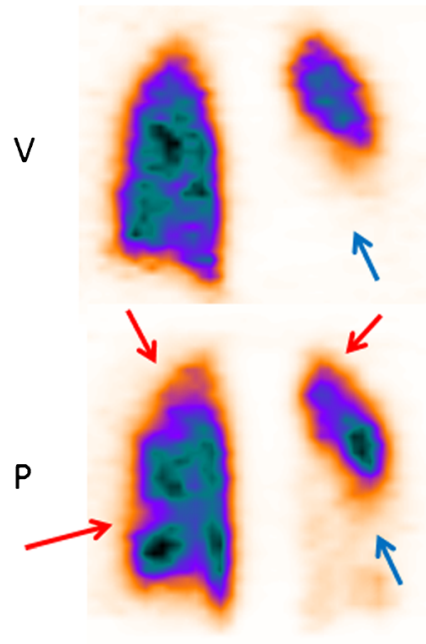

b

Right sagittal slices

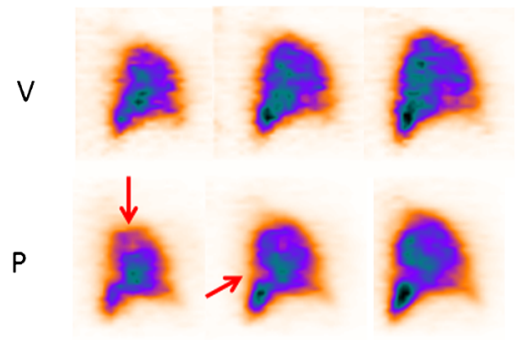

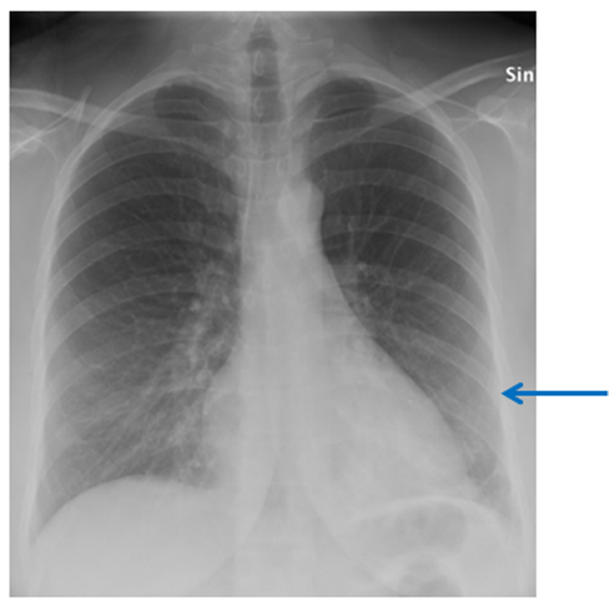

Left sagittal slices

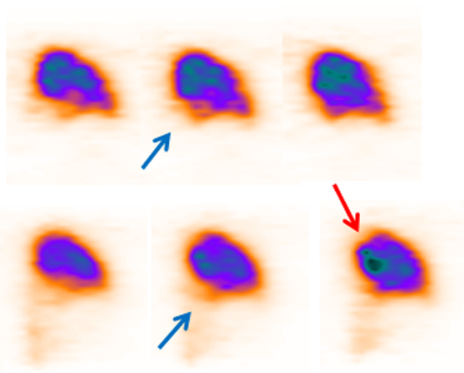

considered pulmonary planar scintigraphy more reliable [19]. Use of planar pulmonary scintigraphy as recommended by some guidelines $[8,9]$ is still often applied in many clinics although it is clearly less sensitive than V/P SPECT [20-23].

In this retrospective study, no V/P SPECT examination in pregnancy was not diagnostic, in contrast to the inconclusive results in 6 of the $61(10 \%)$ women undergoing MDCT during the same period. Such a good performance is in line with other studies that have evaluated V/P SPECT for PE diagnosis in larger cohorts of non-pregnant patients [22, 24-27].

The debate regarding the preferred method of PE diagnosis in pregnancy is still ongoing. Guidelines in general recommend pulmonary scintigraphy as a first choice method $[6,8$,
9]. However, American guidelines [9] recommend an MDCT if the chest X-ray is pathological. This is probably because planar scintigraphy traditionally has been used by the majority of institutions until now. The results of this study of pregnant patients and other studies in patients with suspected PE in general showed that a pathological X-ray is not a contraindication to the use of V/P SPECT. European guidelines, by contrast, recommend pulmonary SPECT as a first choice regardless of pathological chest X-ray [6].

In addition to sensitivity, one important reason supporting the use of V/P SPECT in pregnancy is safety, both for the fetus and the pregnant woman. The measured breast absorbed dose with V/P SPECT was $0.8 \mathrm{mGy}$ in our study. This is a low

Table 4 Fetal absorbed dose (mGy) calculated to the stage of gestation after i.v. injection of ${ }^{99 \mathrm{~m}} \mathrm{Tc}-\mathrm{MAA}$ and inhalation of ${ }^{99 \mathrm{~m}} \mathrm{Tc}-\mathrm{Technegas}$ in 127 pregnant women undergoing V/P SPECT for suspected PE

\begin{tabular}{llll}
\hline Stage of gestation & $\begin{array}{l}\text { Absorbed dose after } \\
50 \mathrm{MBq}{ }^{99 m} \mathrm{Tc}-\mathrm{MAA}\end{array}$ & $\begin{array}{l}\text { Absorbed dose after } \\
30 \mathrm{MBq}{ }^{99 \mathrm{~m}} \mathrm{Tc}-\mathrm{Tech} \text { egas }\end{array}$ & $\begin{array}{l}\text { Absorbed dose after 120 } \\
\mathrm{MBq}{ }^{99 \mathrm{~m}} \mathrm{Tc}-\mathrm{MAA}\end{array}$ \\
\hline Early & 0.14 & 0.007 & 0.34 \\
3 months & 0.20 & 0.007 & 0.48 \\
6 months & 0.25 & 0.011 & 0.60 \\
9 months & 0.20 & 0.014 & 0.48 \\
\hline
\end{tabular}


figure in comparison to calculated breast absorbed doses of 20-50 mGy reported for MDCT [28-33]. Fetal absorbed doses, on the other hand, are similar for MDCT and V/P SPECT [29].

When choosing a diagnostic method, it is always important to assess its negative predictive value. None of the women in whom the P SPECT or V/P SPECT examination was negative for PE was diagnosed with PE or DVT later during the same pregnancy or the following puerperal period, confirming that the method performs well also in this respect. The V/P SPECT examination is thus a fast, safe and reliable way of excluding $\mathrm{PE}$ in this important patient group.

\section{Conclusion}

This study showed that among pregnant women with suspected disease the incidence of a positive scan suggesting $\mathrm{PE}$ is low (9\%), reaffirming the need for low radiation doses. The negative predictive value of V/P SPECT was high, and the radiation exposure from V/P SPECT was low, both for the fetus and the patient. A ventilation study was helpful to clarify patient symptoms and might be cost-effective regarding time and need for other examinations.

Acknowledgments This study was supported by grants from the Research Funds at Skåne University Hospital (SUS fonder) and the Swedish State under the LUA/ALF agreement.

Compliance with ethical standards

\section{Conflicts of interest None.}

Statement of human rights: all procedures performed in studies involving human participants were in accordance with the clinical standards and the institutional and/or national research committee and with the 1964 Declaration of Helsinki and its later amendments or comparable ethical standards.

This study does not contain any studies with animals performed by any of the authors. This is a retrospective study: "For this type of study formal consent is not required."

Open Access This article is distributed under the terms of the Creative Commons Attribution 4.0 International License (http:// creativecommons.org/licenses/by/4.0/), which permits unrestricted use, distribution, and reproduction in any medium, provided you give appropriate credit to the original author(s) and the source, provide a link to the Creative Commons license, and indicate if changes were made.

\section{References}

1. Henriksson P, Westerlund E, Wallén H, Brandt L, Hovatta O, Ekbom A. Incidence of pulmonary and venous thromboembolism in pregnancies after in vitro fertilisation: cross sectional study. BMJ 2013;346:e8632.

2. Virkus RA, Løkkegaard EC, Lidegaard Ø, Langhoff-Roos J, Bjerregaard L, Skovlund CW, et al. Venous thromboembolism in pregnancy and the puerperal period: a study of 1210 events. Acta Obstet Gynecol Scand 2013;92(10):1135-42.
3. Gherman RB, Goodwin TM, Leung B, Byrne JD, Hethumumi R, Montoro M. Incidence, clinical characteristics, and timing of objectively diagnosed venous thromboembolism during pregnancy. Obstet Gynecol 1999;94(5 Pt 1):730-4.

4. Cutts BA, Tran HA, Merriman E, Nandurkar D, Soo G, DasGupta $\mathrm{D}$, et al. The utility of the Wells clinical prediction model and ventilation-perfusion scanning for pulmonary embolism diagnosis in pregnancy. Blood Coagul Fibrinolysis 2014;25(4):375-8.

5. Sivandarajah S. Towards evidence-based emergency medicine: best BETs from the Manchester Royal Infirmary. BET 4: current evidence does not support the use of a negative D-dimer to rule out suspected pulmonary embolism in pregnancy. Emerg Med J 2011;28(3):245-6.

6. Bajc M, Neilly JB, Miniati M, Schuemichen C, Meignan M, Jonson B. EANM guidelines for ventilation/perfusion scintigraphy: part 2 . Algorithms and clinical considerations for diagnosis of pulmonary emboli with V/P(SPECT) and MDCT. Eur J Nucl Med Mol Imaging 2009;36(9):1528-38.

7. Bajc M, Neilly JB, Miniati M, Schuemichen C, Meignan M, Jonson B et al. EANM guidelines for ventilation/perfusion scintigraphy: part 1. Pulmonary imaging with ventilation/perfusion single photon emission tomography. Eur J Nucl Med Mol Imaging 2009;36(8):1356-70.

8. Konstantinides SV, Torbicki A, Agnelli G, Danchin N, Fitzmaurice D, Galiè N, et al. 2014 ESC Guidelines on the diagnosis and management of acute pulmonary embolism. Eur Heart J 2014;35(43):3033-69.

9. Leung AN, Bull TM, Jaeschke R, Lockwood CJ, Boiselle PM, Hurwitz LM, et al. American Thoracic Society documents: an official American Thoracic Society/Society of Thoracic Radiology Clinical Practice Guideline-evaluation of suspected pulmonary embolism in pregnancy. Radiology 2012;262(2):635-46.

10. ICRP. Radiation dose to patients from radiopharmaceuticals, publication 53. Oxford: ICRP; 1988.

11. ICRP. Radiation dose to patients from radiopharmaceuticals, publication 80 (addendum to ICRP 53). Ann ICRP 1998;28(3).

12. Russell JR, Stablin MG, Sparks RB, Watson E. Radiation absorbed dose to the embryo/fetus from radiopharmaceuticals. Health Phys 1997;73:756-69.

13. Palmer J, Bitzén U, Jonson B, Bajc M. Comprehensive ventilation/ perfusion SPECT. J Nucl Med 2001;42(8):1288-94.

14. Bajc M, Olsson C-G, Palmer J, Jonson B. Quantitative ventilation/ perfusion SPECT $\left(\mathrm{QV} / \mathrm{P}_{\mathrm{SPECT}}\right)$ : a primary method for diagnosis of pulmonary embolism. In: Freeman ML, editor. Nuclear medicine annual. Philadelphia: Lippincott Williams \& Wilkins; 2004. p. 173.

15. Bajc M, Markstad H, Jarenbäck L, Tufvesson E, Bjermer L, Jögi J. Grading obstructive lung disease using tomographic pulmonary scintigraphy in patients with chronic obstructive pulmonary disease (COPD) and long-term smokers. Ann Nucl Med 2015;29(1):91-9.

16. Pahade JK, Litmanovich D, Pedrosa I, Romero J, Bankier AA, Boiselle PM. Quality initiatives: imaging pregnant patients with suspected pulmonary embolism: what the radiologist needs to know. Radiographics 2009;29(3):639-54.

17. Shahir K, Goodman LR, Tali A, Thorsen KM, Hellman RS. Pulmonary embolism in pregnancy: CT pulmonary angiography versus perfusion scanning. AJR Am J Roentgenol 2010;195(3): W214-20.

18. Stein PD, Fowler SE, Goodman LR, Gottschalk A, Hales CA, Hull $\mathrm{RD}$, et al. Multidetector computed tomography for acute pulmonary embolism. N Engl J Med 2006;354(22):2317-27.

19. Ridge CA, McDermott S, Freyne BJ, Brennan DJ, Collins CD, Skehan SJ. Pulmonary embolism in pregnancy: comparison of pulmonary CT angiography and lung scintigraphy. AJR Am J Roentgenol 2009;193(5):1223-7.

20. Bajc M, Olsson CG, Olsson B, Palmer J, Jonson B. Diagnostic evaluation of planar and tomographic ventilation/perfusion lung images in patients with suspected pulmonary emboli. Clin Physiol Funct Imaging 2004;24(5):249-56. 
21. Gutte H, Mortensen J, Jensen CV, von der Recke P, Petersen CL, Kristoffersen US, et al. Comparison of V/Q SPECT and planar V/Q lung scintigraphy in diagnosing acute pulmonary embolism. Nucl Med Commun 2010;31(1):82-6.

22. Quirce R, Ibáñez-Bravo S, Jiménez-Bonilla J, Martínez-Rodríguez I, Martínez-Amador N, Ortega-Nava F, et al. Contribution of V/Q SPECT to planar scintigraphy in the diagnosis of pulmonary embolism. Rev Esp Med Nucl Imagen Mol 2014;33(3):153-8.

23. Reinartz P, Schirp U, Zimny M, Sabri O, Nowak B, Schäfer W, et al. Optimizing ventilation-perfusion lung scintigraphy: parting with planar imaging. Nuklearmedizin 2001;40(2):38-43.

24. Bajc M, Olsson B, Palmer J, Jonson B. Ventilation/perfusion SPECT for diagnostics of pulmonary embolism in clinical practice. J Intern Med 2008;264(4):379-87.

25. Grüning T, Drake BE, Farrell SL, Nokes T. Three-year clinical experience with VQ SPECT for diagnosing pulmonary embolism: diagnostic performance. Clin Imaging 2014;38:831-5.

26. Gutte H, Mortensen J, Jensen CV, Johnbeck CB, von der Recke P, Petersen CL, et al. Detection of pulmonary embolism with combined ventilation-perfusion SPECT and low-dose CT: head-to-head comparison with multidetector CT angiography. J Nucl Med 2009;50(12):1987-92.

27. Leblanc M, Leveillée F, Turcotte E. Prospective evaluation of the negative predictive value of V/Q SPECT using $99 \mathrm{mTc}-T e c h n e g a s$. Nucl Med Commun 2007;28(8):667-72.
28. Astani SA, Davis LC, Harkness BA, Supanich MP, Dalal I. Detection of pulmonary embolism during pregnancy: comparing radiation doses of CTPA and pulmonary scintigraphy. Nucl Med Commun 2014;35(7):704-11.

29. Hurwitz LM, Yoshizumi T, Reiman RE, Goodman PC, Paulson EK, Frush DP, et al. Radiation dose to the fetus from body MDCT during early gestation. AJR Am J Roentgenol 2006;186(3):871-6.

30. Hurwitz LM, Yoshizumi TT, Goodman PC, Nelson RC, Toncheva G, Nguyen GB, et al. Radiation dose savings for adult pulmonary embolus 64-MDCT using bismuth breast shields, lower peak kilovoltage, and automatic tube current modulation. AJR Am J Roentgenol 2009;192(1):244-53.

31. Hurwitz LM, Yoshizumi TT, Reiman RE, Paulson EK, Frush DP, Nguyen GT, et al. Radiation dose to the female breast from 16MDCT body protocols. AJR Am J Roentgenol 2006;186(6): $1718-22$.

32. Nyman U, Bajc M, Eriksson H, Geijer H, Gunnarsson M, Hellgren $\mathrm{M}$, et al. Diagnostik av Lungembolism under graviditet. Skintigrafi eller datortomografi? (Diagnosis of pulmonary embolism in pregnant women. Scintigraphy or computer tomography?). Lakartidningen 2010;107:989-94.

33. Parker MS, Hui FK, Camacho MA, Chung JK, Broga DW, Sethi NN. Female breast radiation exposure during CT pulmonary angiography. AJR Am J Roentgenol 2005;185(5):1228-33. 